\title{
A framework to reconcile frequency scaling measurements, from intracellular recordings, local-field potentials, up to EEG and MEG signals
}

\author{
Claude Bedard, Jean-Marie Gomes, Thierry Bal and Alain Destexhe
}

UNIC, CNRS, Gif sur Yvette, France.

Submitted to the special issue on Extracellular Space (Edited by Reinoud Maex).

(Dated: November 11, 2018)

\begin{abstract}
In this viewpoint article, we discuss the electric properties of the medium around neurons, which are important to correctly interpret extracellular potentials or electric field effects in neural tissue. We focus on how these electric properties shape the frequency scaling of brain signals at different scales, such as intracellular recordings, the local field potential (LFP), the electroencephalogram (EEG) or the magnetoencephalogram (MEG). These signals display frequency-scaling properties which are not consistent with resistive media. The medium appears to exert a frequency filtering scaling as $1 / \sqrt{f}$, which is the typical frequency scaling of ionic diffusion. Such a scaling was also found recently by impedance measurements in physiological conditions. Ionic diffusion appears to be the only possible explanation to reconcile these measurements and the frequency-scaling properties found in different brain signals. However, other measurements suggest that the extracellular medium is essentially resistive. To resolve this discrepancy, we show new evidence that metal-electrode measurements can be perturbed by shunt currents going through the surface of the brain. Such a shunt may explain the contradictory measurements, and together with ionic diffusion, provides a framework where all observations can be reconciled. Finally, we propose a method to perform measurements avoiding shunting effects, thus enabling to test the predictions of this framework.
\end{abstract}

PACS numbers:

\section{INTRODUCTION}

The electric nature of the extracellular medium around neurons is of high importance to correctly interpret the extracellular potentials, such as the local field potential (LFP), as well as more remote potentials, such as the electro-encephalogram (EEG). This electric nature can be captured by appropriate measurements of the extracellular impedance. However, the measurements available today, and their interpretation, are contradictory. While some measurements suggest that the extracellular medium is essentially resistive [1, 2], other measurements [3-6] suggest that the medium is non-resistive, and strongly frequency dependent. There is presently no consensus on this electric nature.

On a theoretical point of view, in the neuronal cable theory initially developed by Rall $[7,8]$, the extracellular medium was assumed to have zero resistance, and the neuron was thus considered as embedded in a supraconductive medium. Later formulations $[9,10]$ included a resistance to represent the medium, but it was always assumed that this resistance is much smaller than that of the membrane. The whole development of cable theory was made under this assumption, and to include non-resistive media in cable equations requires to re-derive the equations from first principles. This was done recently, leading to the "generalized cable theory" [11, 12], that provided a cable theory valid for arbitrarily complex extracellular media (and includes Rall's cable theory as a particular case). It was found that the extracellular impedance appears in the length constant of the neuron, and thus the nature of the medium potentially can influence the integrative properties of neurons [11].

Modeling complex extracellular media started with an initial model that only considered the impedance inhomogeneities (such as fluids and membranes), and it was found that such inhomogeneous structure can lead to strong frequency filtering effects [13-15]. It was later shown, using a mean-field formalism, that various physical processes such as polarization [14,16] or ionic diffusion [14, 17] can similarly cause frequency filtering, and thus influence the genesis of the LFP. It was shown that a medium with polarization is equivalent to a resistance-capacitance circuit [16], thus exerting strong low-pass filtering on extracellular potentials. Similarly, a medium with diffusive properties will also exert a low-pass filtering. None of such filtering is present with a resistive medium.

The nature of the medium can also influence the estimation of neuronal sources from extracellular recordings, thus affecting methods such as the Current Source Density (CSD) analysis [17]. Like cable equations, the CSD method assumes a resistive medium [18], and is not valid for more complex extracellular properties. The CSD method was generalized by rederiving the equations from first principles, yielding a generalized CSD which includes the classic CSD as a particular case, and which can estimate neuronal sources within a non-resistive extracellular medium [17]. 
Here again, it was found that the nature of the medium has potentially large influences on the CSD estimates of neuronal sources.

This underlines the importance of having a precise estimate of the impedance of the extracellular medium. In the present paper, we review a number of measurements at different scales, from intracellular, to LFP, and up to large scale such as the EEG. We also provide new analyses of experimental results and propose a framework where all contradictory data can be explained.

\section{RESULTS}

We first review evidence that different brain signals, such as intracellular recordings, the local field potential (LFP), the electroencephalogram (EEG) or the magnetoencephalogram (MEG), all display properties that are not consistent with resistive media. We next show results from extracellular metal-electrode measurements that suggest a way to resolve discrepancies between different measurements in the literature.

\section{Frequency scaling of different brain signals}

\section{$1 / f$ scaling of EEG and LFP}

EEG and LFP signals can show $1 / f$ frequency scaling properties at low frequencies $(<10 \mathrm{~Hz})$, as illustrated in Fig. 1. This was shown by a number of studies [19-23]. It is important to note here that such $1 / f$ scaling depends on brain state and is seen in awake subjects with strictly desynchronized EEG. In other brain states, the frequency scaling may be different, for example during anesthesia the EEG scales as $1 / f^{2}$ [24]. The frequency scaling illustrated here in the human EEG and in the LFP recorded in cat parietal cortex was done in subjects that were awake and attentive. The exact value of the exponent also varies as a function of the brain region considered, as also shown previously [23].

\section{LFP-unit measurements}

Different mechanisms were proposed to explain the origin of such " $1 / f$ noise" in the brain. $1 / f$ spectra can result from self-organized critical states [25], suggesting that neuronal activity may be working according to such states [26], but this subject is controversial [22, 27]. The morphology of the neuron may also be responsible for filtering in the $1 / f$ to $1 / f^{2}$ range [28], but this scaling applies to high frequencies and cannot explain the
EEG (Human)
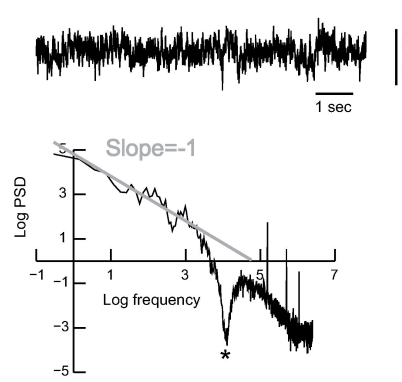

LFP (Cat)
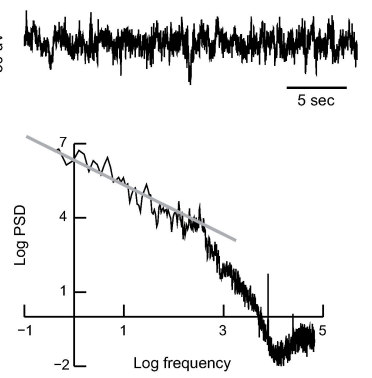

FIG. 1: $1 / f$ Frequency scaling of EEG and LFP signals in awake subjects. Top: human EEG recording (left, vertex EEG) and LFP recording from cat parietal cortex (right) in awake and attentive subjects (desynchronized EEG). Bottom: the corresponding power spectra display approximate $1 / f$ scaling at low frequencies. The straight lines (gray) indicate a slope of -1 (log-log representation). The signals were not filtered, except for a notch filter at $60 \mathrm{~Hz}\left(^{*}\right)$ for the EEG. All power spectra were computed using the Fast Fourier Transform (FFT) algorithm, and were not normalized.

$1 / f$ scaling at low frequencies. Finally, the $1 / f$ scaling may be due to filtering properties of the currents through extracellular media [22]. This conclusion was reached by noting that the global activity reconstructed from multisite unit recordings scales identically as the LFP if a " $1 / \mathrm{f}$ filter" is assumed, and without the need to assume selforganized critical states in neural activity (Fig. 2). However, the latter study made the point that $1 / f$ filtering may be necessary to explain the experimental results, but no mechanism was provided. We will show below that ionic diffusion can explain such a $1 / f$ filter.

\section{Modeling the $1 / f$ scaling of LFPS}

$1 / f$ scaling in power spectra is not easy to explain, because it predicts a filter in $1 / \sqrt{f}$. Classic filters such as a capacitive filter, or an RC-circuit such as in neuronal membranes, would predict $1 / f^{2}$ filtering in power spectra. It was shown that ionic diffusion can generate frequency scaling as $1 / \sqrt{f}[14,29,30]$. Using a macroscopic modeling approach based on a mean-field formulation of Maxwell equations[14, 17], it was shown that ionic diffusion can give rise to $1 / f$ frequency scaling at low frequencies (Fig. 3). This scaling arises because the ionic diffusion in the extracellular medium and around the current sources is responsible for a "diffusion impedance" scaling as $1 / \sqrt{f}$, which gives $1 / f$ in the power spectrum. For high frequencies, the natural $1 / f^{2}$ scaling of current sources (which are mostly exponential) is also subject to the same filter, which gives the observed $1 / f^{3}$ scaling. With ionic diffusion, one can 

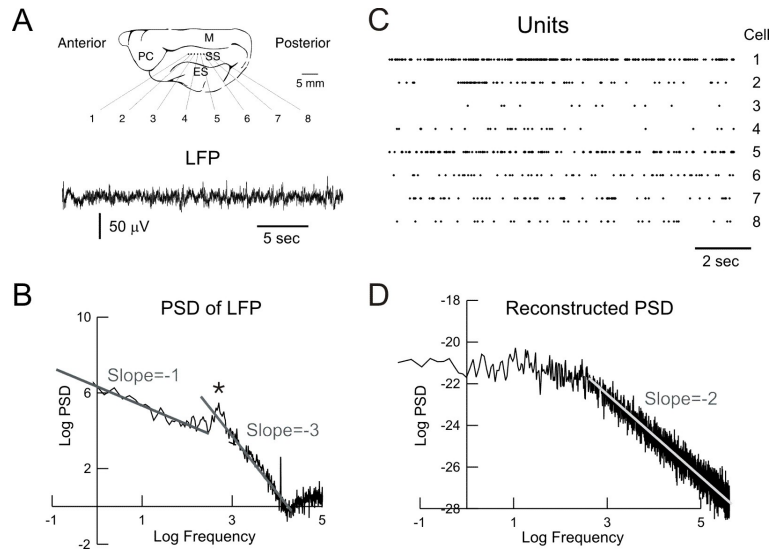

FIG. 2: Relationship between unit activity and LFP power spectra. A. LFP recording in awake cat parietal cortex (same recording as in Fig. 1, right). The top scheme shows the location of the electrodes in parietal cortex. B. Power spectral density (PSD) of the LFP, showing that low frequencies scale as $1 / f$ (gray line, slope $=-1$ ), and $1 / f^{3}$ at higher frequencies (gray line; slope $=-3$ ). C. Unit activity from the same experiment, recorded with a system of 8 tungsten electrodes (schematized in A). D. Attempt to reconstruct the LFP signal from the unit activity. The low frequency end of the PSD was constant (zero slope), while the highfrequency end scaled as $1 / f^{2}$ (gray line, slope $=-2$ ). An exponent of -1 is missing to reproduce the LFP scaling, which could be the sign that the current sources are subject to of an $1 / f$ filter (modified from Bedard et al [22].

qualitatively reconstruct the frequency scaling of LFPs from the unit activity, and thus, ionic diffusion appears as a physically plausible explanation for the observed scaling.

\section{Intracellular-LFP measurements}

To further probe the LFP signal, we used simultaneous intracellular and LFP measurements, as schematized in Fig. 4A. In particular, it is interesting to focus on the transfer function between intracellular and extracellular signals. This transfer function was evaluated from simultaneous intracellular and LFP measurements in rat barrel cortex in vivo [31] and is represented in Fig. 4B (gray). The interest of this approach is that when relating intracellular and extracellular voltages, the impedance of the extracellular medium naturally appears, so matching different models to the measured transfer function allows one to estimate the extracellular impedance. This estimate is indirect, however, because this model is valid only for brain states where neuronal activities are perfectly decorrelated. This is why this estimate must be performed in desynchronized-EEG brain states, as done in Bedard et al [31]. Different hypotheses about the extracellular impedance are shown in Fig. 4 (black curves). Neither resistive nor capacitive media provided acceptable fits, while the
A

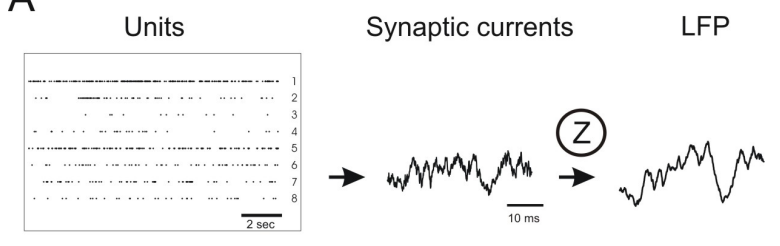

B

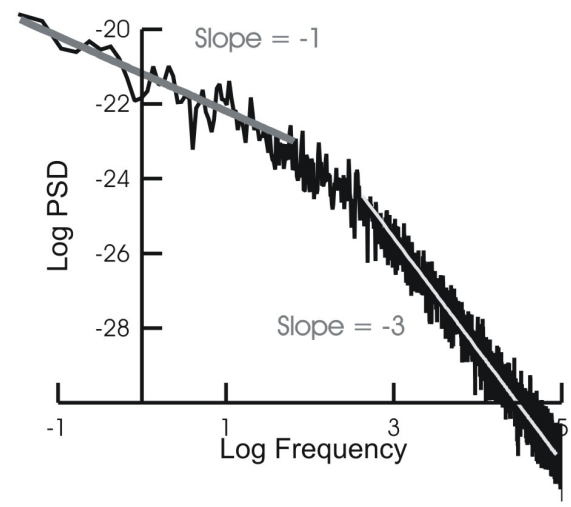

FIG. 3: Reconstruction of LFP power spectra from unit activity using ionic diffusion. A. Scheme of the reconstruction. The unit activity is used to generate a synaptic current. The current is used in a model of LFP that uses a diffusion impedance $(Z$, varying as $1 / \sqrt{(} \omega))$. B. PSD of the modeled LFP, which qualitatively displays the same frequency scaling as the real LFP (compare with Fig. 2B; modified from Bedard and Destexhe, [14]).
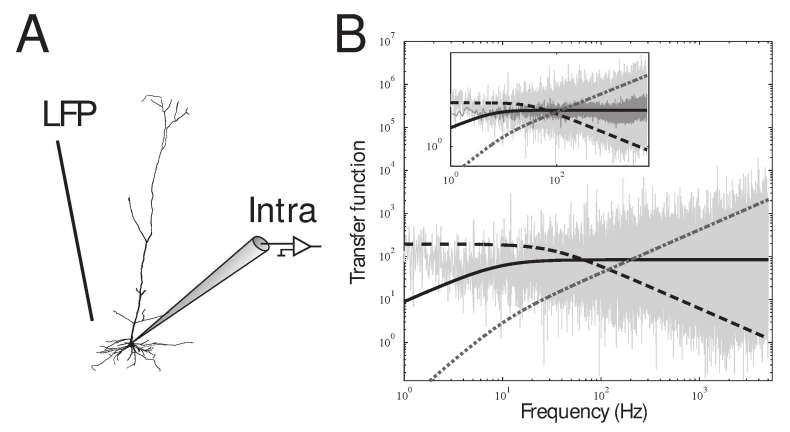

FIG. 4: Transfer function between intracellular and extracellular potentials in vivo. A. Scheme of the recording, where intracellular and LFP measurements are made within a close vicinity. B. Transfer function spectrum computed from a cell recorded in rat barrel cortex in vivo during desynchronized EEG states (gray spectrum). The black lines show different transfer functions calculated from a ball-and-stick model surrounded by media with different impedances, resistive (dashed), diffusive (solid), and capacitive (dotted). Modified from Bedard et al.,[31].

best match was obtained for an impedance scaling as $1 / \sqrt{f}$, compatible with ionic diffusion. Thus, similar to the spectral analysis of LFPs, the LFPintracellular relation is also compatible with an electrical impedance with strong ionic diffusion effects. 


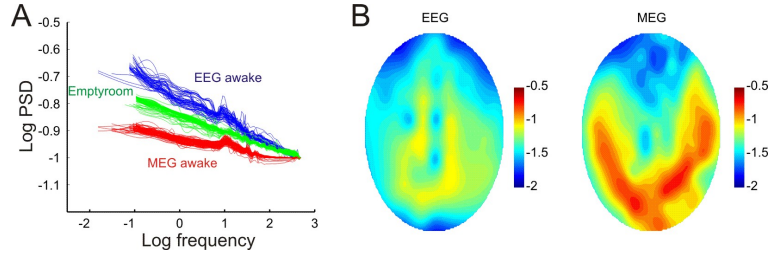

FIG. 5: Different frequency scaling of EEG and MEG signals. A. Frequency spectra of simultaneouslyrecorded EEG (blue) and MEG (red) signals from an awake subject with desynchronized EEG. The "emptyroom" MEG signal (green) is also shown for comparison. B. Left: Distribution of low-frequency scaling exponents over the scalp for the EEG, showing that the low-frequency scaling exponent is comprised mostly between 1 and 2. Right: Same representation for MEG signals. In this case, the exponent is lower, and generally smaller than 1. Modified from Dehghani et al. [23].

\section{EEG-MEG measurements}

Another type of signal that can be used to infer the nature of extracellular space is the magnetic field generated by neuronal activities. In particular, using simultaneously-recorded EEG and MEG signals, it is possible to relate their frequency scaling properties. Theoretical work shows that, if the extracellular medium is resistive, the scaling of EEG and MEG signals at low frequencies should be the same [23]. Similarly to above for LFP and intracellular signals, this relation is only valid assuming that the synaptic inputs are uncorrelated, so it should be evaluated in brain states as desynchronized as possible. The measurement of the frequency scaling in awake human subjects (with desynchronized EEG) showed that the frequency scaling is generally not the same between EEG and MEG signals (Fig. 5). The difference is evident by visual inspection of superimposed spectra (Fig. 5A), and this difference is confirmed by the distribution of scaling exponents in different brain regions (Fig. 5, B). A detailed analysis showed that when the exponents were similar, the signal to noise ratio was very low, and that this difference is significant [23]. Thus, the relation between EEG and MEG signals suggests that the extracellular medium is not resistive, although this analysis does not say more about which type of medium is the most likely.

\section{Intracellular measurements}

Finally, the measurement of the extracellular impedance can be done directly using two micropipettes, as illustrated in Fig. 6 (top scheme). Here, the intracellular recording was performed in reference to a nearby micropipette in the extracellular medium, and a subthreshold white noise current input was injected into the cell. The re- lationship between the injected current, and the difference between intracellular and extracellular voltages, gives a direct access to the extracellular impedance. This measurement is done here in natural conditions, because no current is injected in the extracellular space, and the amount of current is also much smaller and is within the physiological range. This is different from the classic metal-electrode measurements of impedance, which must use artificially high currents, and also involve a complex interface between the metal and the living medium. This "natural" impedance measurement [6] is therefore more accurate and more physiological because the current source in the medium is the neuron itself, using all the natural biochemical and biophysical mechanisms of how cells interact with the surrounding medium.

It is important to note that this measurement is very different from that of Fig. 4, although both involve simultaneous intracellular and extracellular recordings. In the case of Fig. 4, the ongoing activity is analyzed, and different guesses for the extracellular impedance are compared to the measured transfer function, whereas in the present case, the impedance is directly measured by controlling the injected current.

An example of the measured impedance for a representative cell is shown in Fig. 6. The measurement of the impedance modulus amplitude (Fig. 6A) and phase (Fig. 6B) show a frequency profile that significantly departs from that predicted by a resistive impedance (blue curves). On the other hand, a diffusion impedance accurately predicts the measured frequency profile (Fig. 6A$\mathrm{B}$, green curves). The same result was also obtained by white-noise current injection in vivo, or by injection of sinusoidal currents in vitro [6].

\section{Metal-electrode measurements}

The results reviewed in the previous section show that the frequency scaling of different brain signals, from microscopic to macroscopic scales, all point to the fact that the medium is well described by a diffusion impedance. However, this result is not in agreement with previous measurements using metal electrodes, suggesting a resistive extracellular medium [2]. To further investigate this issue, we have performed additional experiments.

Using the same setup as schematized in Fig. 6, it is also possible to measure the transfer function of the system, as illustrated in Fig. 7A-B. In this case, we have fit the measured function with the same models as before, a resistive and a diffusive model, as shown by the blue and green curves in Fig. 7A-B, respectively. Similar to above, the dif- 


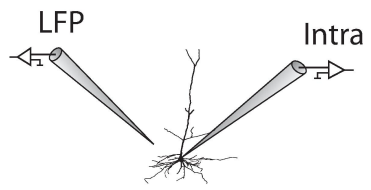

A

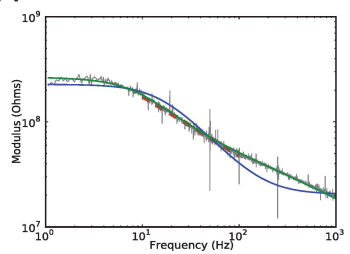

B

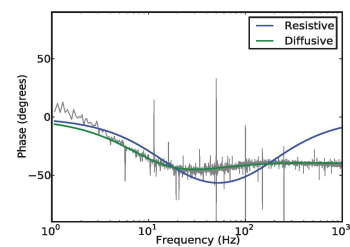

FIG. 6: Natural impedance measurement in vitro. Top: experimental setup, where a subthreshold white-noise current was injected in a cell in cortical slices, together with an extracellular recording in the vicinity using a second micropipette located about $20 \mu \mathrm{m}$ away from the soma of the patched cell. The modulus (A) and phase (B) of the measured impedance are shown as a function of frequency. The colored curves show the best fits using a resistive model (blue) and a diffusive model (green). Modified from Gomes et al. [6].
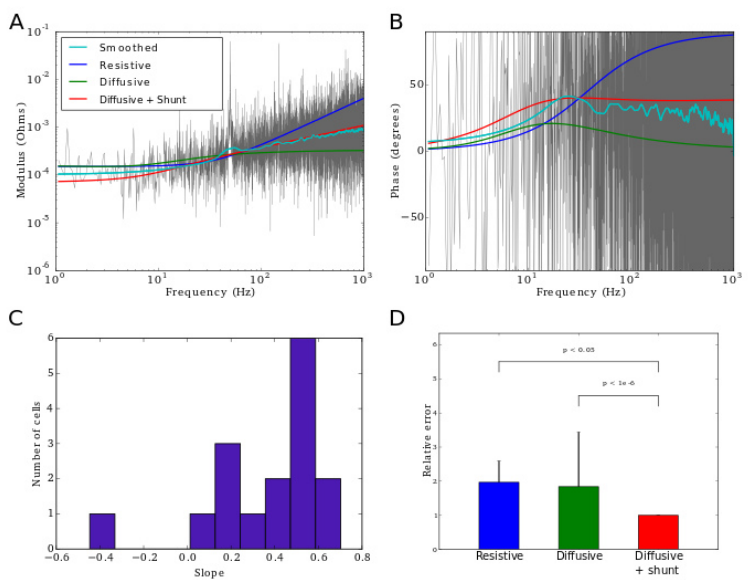

D

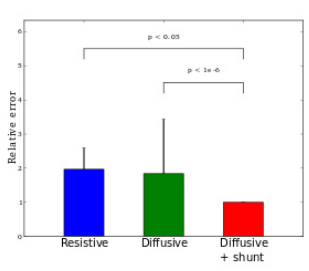

FIG. 7: Transfer function between intracellular and extracellular potentials in vitro. A: Modulus of the transfer function $V_{L F P} / V_{\text {intra }}$ as a function of frequency (gray). B. Same as in A, but for the phase. In A and B, the colored curves are respectively: resistive model (blue), diffusive model (green) and a model including ionic diffusion and a possible shunt in the measurement (red). C. Distribution of the frequency scaling exponent found for different cells. D. Relative error of different models with respect to the data.

fusive model provided a better fit of the transfer function, but the difference was essentially due here to the phase of the transfer function (whereas in Fig. 4, only the PSD was shown).

To better explain these results, we made the following hypothesis, as schematized in Fig. 8A. Metal electrodes, due to their large diameter (microns), are necessarily surrounded by a thin layer of cerebro-spinal fluid (CSF; also called artificial CSF or ACSF in the slice), and thus when injecting currents in a metal electrode, part of the current flows through the tissue, but another part of the current may also flow through the CSF (red
A

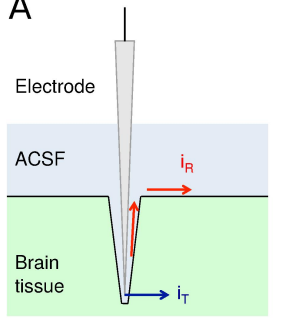

B

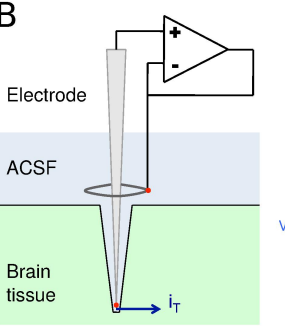

C

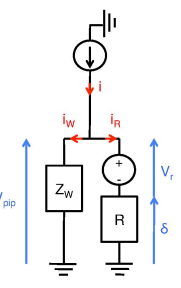

FIG. 8: Experiment to test the presence of a shunt. A. When an electrode is inserted in brain tissue to inject a current, this current flows in the tissue $\left(i_{T}\right)$ and in a nearly-resistive fluid layer (ACSF) flowing through the surface $\left(i_{R}\right)$. B. Setup where a voltage follower imposes to the surface the same potential as that of the tip of the electrode (red dots). This prevents currents from flowing in the fluid layer and forces the current to flow into the brain tissue. C. Equivalent circuit of this setup. The round shapes with an arrow and +/- signs are respectively current and voltage sources.

arrow in Fig. 8A), thereby creating a shunt. Such a shunt will necessarily be resistive because the current flows only through the CSF liquid. To test whether such a hypothesis is plausible, we included a resistive shunt in parallel to the diffusive impedance. Such a "diffusive + shunt" model was able to better fit the measured transfer function (red curves in Fig. 7). In particular, the error was much smaller by using such a shunt (Fig. 7D).

This measurement and fitting suggest that previous metal electrode measurements may give the impression that the medium is resistive, because part of the current goes through the CSF. This situation was examined in more detail in the Appendix, where we show that the measured impedance will be a combination of the tissue impedance and the impedance of the CSF, so all depends on the ratio of currents that flow in each medium.

Finally, we would like to propose a way to experimentally avoid this shunting effect through the CSF, as illustrated in Fig. 8B-C. To avoid that current flows through the CSF, one could use another electrode and a voltage-follower circuit to clamp the voltage at the surface to the same value as that of the tip of the electrode. This additional electrode could take the form of an ring around the metal electrode. This way, because the surface and the tip will be at the same voltage, there will be no current flow through the CSF, and this should force the current to flow through the extracellular medium.

\section{DISCUSSION}

In this paper, we have reviewed different measurements, from single neurons to large-scale recordings, which all converge to the same con- 
clusions: (1) the extracellular medium around neurons cannot be considered as purely resistive; (2) all results can be explained assuming a frequency filter scaling as $1 / \sqrt{f}$; (3) ionic diffusion appears as the physical process that explains most of these results. This includes the correlation between single-cells and LFPs, both at the level of the transfer function [31], and direct impedance measurements [6]. It also accounts for the $1 / f$ scaling of LFPs, and its relation with the unit activity [22], as well as for the relation between EEG and MEG signals, that scale differently at low frequencies [23].

However, although these results cumulate into a quite strong evidence, they do not constitute a proof that the medium is diffusive. This is in part because most of these results were obtained in ongoing-activity conditions, where multiple sources were present in the neuron, and were not controllable. An exception is the impedance measurement in vitro [6], where a single source was present and controlled. In this case, the current source was known, as well as the intracellular and extracellular voltage, and their modulus and phase relations showed particular frequency profiles, that only ionic diffusion was able to capture. It may be that taking into account the dendritic filtering effect [28] accounts for parts of these results as well (T. Ness and G. Einevoll, private communication). However, it was found that the dendritic filtering effect vanishes under in vivo conditions (see Fig. 2 in [31]), and the same measurements were also obtained in vivo [6], which suggests that dendritic filtering is not a likely explanation for those results. In addition, dendritic filtering does not explain the low-frequency scaling, nor the difference of scaling between EEG and MEG signals, and it was shown explicitly [31] that it cannot account for the transfer function measurements in vivo.

A main prediction from the impedance measurements is that the extracellular impedance is of the same order - or even larger - than that of the cell membrane, contrary to previous measurements. This is completely opposed to the classic view of a very low extracellular impedance, which is usually neglected in the cable formalism for modeling neurons $[7,8,10]$. According to this classic view, the extracellular medium is a bypass, even considered with zero resistance (supraconductive) in some cable formalisms .

In the present paper, we provided a first test of this prediction. By using experiments with two micropipettes [6], one intracellular (wholecell) and one extracellular, we could evaluate the transfer function in vitro $\left(V_{L F P} / V_{\text {intra }}\right)$ (see Fig. 7). This experiment shows that the difference of impedance as estimated from these two electrodes is very small, which confirms the prediction.
To provide a plausible way to resolve the discrepancy between these experiments and previous measurements (e.g., [2]), we proposed a possible explanation based on a resistive current shunt via the CSF on the surface of the brain (or ACSF in the slice), as schematized in Fig. 8A. This resistive shunt, combined with ionic diffusion, provides a better fit of the measured transfer function (Fig. 7), but the improvement of the fit is not by itself a proof of the existence of such a shunt, so it remains a prediction. It does provide an explanation for why some of the measurements of extracellular impedance concluded on a resistive medium. We hypothesize that, in these measurements, the part of the current flowing through the CSF was large, so that the high impedance of the extracellular medium was basically invisible. In addition to proposing this shunt hypothesis, we also suggested a method to evaluate this effect experimentally (Fig. 8). We hope that further experiments will use that method in order to clarify the issue and explain the contradictory measurements.

Why is the extracellular medium characterized by a diffusive impedance? There are currently two - non exclusive - possible theoretical explanations. The first possible explanation is that ionic diffusion acts at the source of the current, in or near the transmembrane ion channels. It is well known that ionic diffusion is central to establish and maintain the membrane potential [32], and ionic diffusion is also necessarily implicated in reequilibrating the ionic concentrations, and maintaining the Debye layer in the vicinity of the membrane. It is thus possible that the visible current source in the extracellular medium contains an important contribution from ionic diffusion, which may explain why this component is seen in the measurements. This of course would consider that the current flows in an essentially resistive extracellular medium.

A second possible explanation does not postulate any special effect of ionic diffusion at the source of the current, but how the current flows in the extracellular medium. The current flow is necessarily associated with an electric field, and the field lines will depend on the charge distribution in the cell, and the flow of charges will follow these field lines. However, the field lines will in general not respect the complex shape of the interstitial space in the extracellular medium. Thus, the charges that follow the field lines will necessarily meet obstacles (such as cell membranes or vessels), and produce local concentration inhomogeneities. Such concentration gradients will implicate ionic diffusion. Thus, ionic diffusion will be the mechanism that will allow the charges to circumvent the obstacles, and this may explain why the impedance is high, and why it has a diffusive component. Future experiments should 
be designed to further test these possible mechanisms.

Finally, it is important to mention that ionic diffusion combined with a shunt, is so far the only coherent framework in which all the experimental measurements find a possible explanation. Other hypotheses, such as the resistive medium or the dendritic filtering, cannot explain some of the data. This of course does not mean that ionic diffusion is the correct framework, but we hope it will motivate further experiments to clarify the exact electrical nature of the extracellular medium.

In conclusion, we have shown that since the classic work on cable equations [7-10] and CSD analysis [18], which all considered that the extracellular medium is resistive, there is quite substantial evidence for deviations from resistivity. If the medium is non resistive, all the above formalisms are invalid and must be re-derived from first principles (Maxwell equations). This was done for cable equations [11] and CSD analysis [17], which were generalized to be valid with arbitrarily complex extracellular media. In the present paper, we review that indeed, there is quite strong evidence that the medium may be diffusive instead of resistive. Thus, we conclude that experiments should now focus on evaluating the possible consequences of such non-resistivity on the integrative properties of single neurons, as well as on the genesis of extracellular potentials.

\section{Acknowledgments}

Research funded by the CNRS, the Paris-Saclay excellence network (IDEX) and the European Community (Human Brain Project, H2020-720270).

\section{Appendices}

Impedance measurement in the presence of a shunt

In this appendix, we show that if there is an extracellular shunt, the measured impedance may be resistive, even with a non-resistive medium.

We start from the scheme in Fig. 9, where the injecting electrode (left) and the reference electrode (right) are in contact with the extracellular medium, as well as with the surrounding CSF. For modeling such a "macroscopic" measurement, one must use a mean-field version of Maxwell equations [17, 33]. According to the quasi-static electric approximation in mean-field, the electric potential is solution of the Laplace equation:

$$
\nabla^{2} V=0
$$

when the size of the volume element considered for the mean-field is sufficiently large. Note that this equation applies to the extracellular medium, as well as to the surrounding CSF, and its solution is unique for given boundary conditions. Also note that this equation is the same in time or Fourier frequency space, because the Laplacian is a linear and time independent operator. In the following, we use this equation in Fourier frequency space for more convenience.

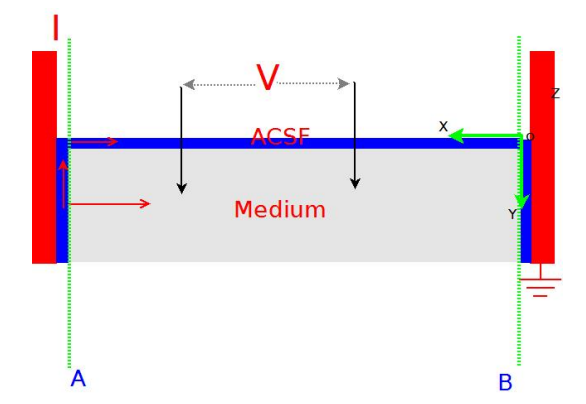

FIG. 9: Scheme of an experimental setup for measuring impedances in biological tissue. The injecting electrode (left) and reference electrode (right) are in contact with both the biological medium (gray) and the liquid layer (ACSF, blue), and thus the current flows in both (red arrows). In a 4-electrode setup, two passive electrodes (middle, black arrows) are inserted in between the injecting and reference electrodes and are used to measure the voltage difference.

We now consider a typical setup to measure the impedance (or admittance) of biological tissue, as schematized in Fig. 9. Our assumption is that the electrode is surrounded by a liquid layer of CSF (schematized in blue), and that part of the current flows through the medium, but also through this layer (red arrows). Assuming that the thickness of the tissue (slice or in vivo) is large compared to the spatial scale of the mean-field, the electric potential must be solution of the 2D Laplace equation:

$$
\frac{\partial^{2} V}{\partial x^{2}}+\frac{\partial^{2} V}{\partial y^{2}}=0
$$

At a very short distance of the current injecting electrodes, we have the following constraint (by symmetry):

$$
\frac{\partial V\left(l_{x}, y\right)}{\partial x}=\frac{\partial V(0, y)}{\partial x}
$$

when the electrodes are identical.

We now solve this system for the region between plane $A$ and $B$ (see Fig. 9). According to the Stone-Weierstrass theorem, the general solution of the 2D Laplace equation on a compact domain can be represented by a two-variable series with integer exponents. One can group the terms of similar degree to form homogeneous polynomials and calculate their coefficients so that they are solution of Laplace equation. 


$$
\left\{\begin{aligned}
P_{0} & =1 \\
P_{1} & =a_{1} x+b_{1} y \\
P_{2} & =a_{2}\left(x^{2}-y^{2}\right)+b_{2} x y \\
P_{3} & =a_{3}\left(x^{3}+3 x y^{2}\right)+b_{3}\left(y^{3}+3 x^{2} y\right) \\
P_{\ldots} & =\cdots
\end{aligned}\right.
$$

This method is equivalent to the construction of the particular solutions of 3D Laplace equation using spherical polynomials [34]. Thus, we can write the solution of Laplace equation (2) as:

$$
V(x, y)=\sum_{i=0}^{\infty} C_{i} P_{i}(x, y) .
$$

The symmetry condition (Eq. 3) implies that $C_{i}=0$ for $i>2$ and $a_{2}=0$, so that the general solution is given by:

$$
\begin{aligned}
V(x, y ; \omega) & =C_{1} a_{1} x+C_{1} b_{1} y+C_{2} b_{2}(x y) \\
& =D_{1} x+D_{2} y+D_{3} x y
\end{aligned}
$$

Note that here, the origin is placed on the reference electrode (see Fig. 9), which implies $V(0,0)=0$, so that we necessarily have $C_{o}=0$ in the expression (5). It follows that the electric field is given by:

$$
\vec{E}=-\nabla V=-\left(D_{1}+D_{3} y\right) \hat{e}_{x}-\left(D_{2}+D_{3} x\right) \hat{e}_{y},
$$

and the respective current densities in CSF and in the medium are given by:

$\left\{\begin{aligned} \vec{j}_{C S F}^{g} & =-\gamma_{C S F}\left[\left(D_{1}+D_{3} y\right) \hat{e}_{x}+\left(D_{2}+D_{3} x\right) \hat{e}_{y}\right] \\ \vec{j}_{\text {medium }}^{g} & =-\gamma_{\text {medium }}\left[\left(D_{1}+D_{3} y\right) \hat{e}_{x}+\left(D_{2}+D_{3} x\right)\right.\end{aligned}\right.$

We can derive the following expression for the current:

$\left\{\begin{aligned} I_{C S F}^{g} & =\gamma_{C S F}\left(D_{1}+\frac{1}{2} D_{3} l_{y}^{C S F}\right) A_{C S F} \\ I_{\text {medium }}^{g} & =\gamma_{\text {medium }}\left(D_{1}+\frac{1}{2} D_{3} l_{y}^{\text {medium }}\right) A_{\text {medium }}\end{aligned}\right.$

where $A_{C S F}$ is the area of the CSF layer along the $Y Z$ plane, and $A_{\text {medium }}$ is the area of the extracellular medium (along the $Y Z$ plane as well). $l_{y}^{C S F}$ is the thickness of the CSF layer, and $l_{y}^{\text {medium }}$ is the thickness of the medium layer.

To keep the formalism as general as possible, and allow the impedance of the extracellular medium to be non-resistive, we use the generalized current conservation law. Applying this current conservation implies that $I_{C S F}^{g}+I_{\text {medium }}^{g}=$ $I^{g}$ does not depend on $x$.

We can now evaluate $D_{1}$ from the potential difference between position $(0,0)$ and $\left(l_{x}, 0\right)$ (see Fig. 9). We have

$$
\Delta V_{A}^{B}(y=0)=-\int_{(0,0)}^{\left(l_{x}, 0\right)} \vec{E} \cdot d \vec{s}=D_{1} l_{x} .
$$

Similarly, we can evaluate $D_{3}$, the potential difference between $\left(0, l_{y}\right)$ and $\left(l_{x}, l_{y}\right)$. We have

$\Delta V_{A}^{B}\left(y=l_{y}\right)=-\int_{\left(0, l_{y}\right)}^{\left(l_{x}, l_{y}\right)} \vec{E} \cdot d \vec{s}=D_{1} l_{x}+D_{3} l_{y} l_{x}$.

Thus, we can write

$$
\left\{\begin{array}{ccc}
D_{1} & =\frac{\Delta V_{A}^{B}(y=0)}{l_{x}} \\
D_{3}= & \frac{\Delta V_{A}^{B}\left(y=l_{y}\right)-\Delta V_{A}^{B}(y=0)}{l_{y} l_{x}}
\end{array}\right.
$$

By assuming

$$
<\Delta V_{A}^{B}>=\frac{1}{2}\left[\Delta V_{A}^{B}(y=0)+\Delta V_{A}^{B}\left(y=l_{y}\right)\right]
$$

we can write (see Eq. 8):

$$
\begin{aligned}
I^{g} & =Y_{e q} \Delta V_{A}^{B}(y=0) \\
& =\left(Y_{C S F}+Y_{\text {medium }}\right) \Delta V_{A}^{B}(y=0) \\
& =\left(\gamma_{C S F} \frac{A_{C S F}}{l_{x}}+\gamma_{\text {medium }} \frac{A_{\text {medium }}}{l_{x}}\right) \Delta V_{A}^{B}(y=0)
\end{aligned}
$$

where we assume that $\left\langle\Delta V_{A}^{B}\right\rangle \approx \Delta V_{A}^{B}$ (y= $0)$, which is equivalent to neglect the electrode impedance. Note that neglecting the electrode impedance augments the ratio of current that goes through the medium, compared to the current that flows through the CSF, and thus this approximation diminishes the shunting effect. $Y_{C S F}$ and $Y_{\text {medium }}$ are the macroscopic admittances of the CSF and extracellular medium, respectively. Note that the "microscopic" admittance is usually called $\gamma$, while $Y$ is the macroscopic admittance, as usually defined in electronics for example. Once the current is fixed, for example by a current source, the knowledge of $Y_{e q}(\omega)$ gives access to $<\Delta V_{A}^{B}>$ et , which allows one to determine $V(x)$ at every point in space. Thus, the measure of the global current and the potential difference between planes $A$ and $B$ does not give information about each macroscopic admittance, but only about a global admittance (the sum of each admittance in the system).

We now examine different possible scenarios for the respective values of these admittances.

Scenario 1: small medium admittance. If we assume that the admittance of the medium is much smaller than that of CSF, we have:

$$
I \approx Y_{C S F} \Delta V_{A}^{B}
$$

Scenario 2: large medium admittance. If the medium admittance is larger than that of CSF, we have:

$$
I \approx Y_{\text {medium }} \Delta V_{A}^{B}
$$


Scenario 3: admittances of comparable magnitude. If we have nearly equal admittances, then we have:

$$
\begin{aligned}
I & =\left(Y_{\text {medium }}+Y_{C S F}\right) \Delta V_{A}^{B} \\
& \approx 2 Y_{\text {medium }} \Delta V_{A}^{B}=2 Y_{C S F} \Delta V_{A}^{B} .
\end{aligned}
$$

Thus, we see that the measured global admittance highly depends on the relative admittance of the medium and CSF. For example, finding a weak frequency dependence of the measurement (as in [2]) may mean that the medium is resistive, but it could also mean that $Y$ medium $>Y_{C S F}$ (Scenario 1). Recent measurements [6] suggest that indeed Ymedium is very high, and the experiments reported here (Fig....) suggest that a significant shunt is present with metal electrodes, so $Y C S F$ is likely to be small. We expect that this admittance will be small for large electrodes and will be larger for small electrodes such as micropipettes.

In a four-electrode measurement setup, from Eq. 2, we have:

$$
V(x)=\frac{I}{Y_{C S F}+Y_{\text {medium }}} \frac{x}{l_{x}}
$$

such that the voltage difference measured by the two central electrode is given by:

$$
\Delta V=\frac{I}{Y_{C S F}+Y_{\text {medium }}} \frac{\Delta x}{l_{x}},
$$

where $\Delta x$ is the distance between the two central electrodes.

We see that with a 4-electrode setup, the measured impedance will also be dependent on the relative values of the admittance of the medium and CSF. Thus, even in such a setup, if a significant fraction of the current flows through the CSF, the impedance of the medium may be invisible in practice. In the main text, we suggest a method to prevent this possible source of error in the measurement.

[1] Ranck, J. (1963) Analysis of specific impedance of rabbit cerebral cortex. Exp. Neurol. 7: 144-152.

[2] Logothetis N.K., Kayser, C. and Oeltermann, A. (2007) In vivo measurement of cortical impedance spectrum in monkeys : implications for signal propagation. Neuron 55: 809-823.

[3] Schwan, H. (1968) Electrode polarization impedance and measurements in biological materials. Ann. New York Acad. Sci. 148: 191-209.

[4] Gabriel, S., Lau, R.W. and Gabriel, C. (1996) The dielectric properties of biological tissues : II. Measurements in the frequency range $10 \mathrm{~Hz}$ to $20 \mathrm{GHz}$. Phys. Med. Biol. 41: 2251-2269.

[5] Nelson M., Bosch, C., Venance, L., and Pouget, P. (2013) Microscale Inhomogeneity of Brain Tissue Distorts Electrical Signal Propagation. J. Neurosci. 33: 3502-3512.
[6] Gomes JM, Bédard C, Valtcheva S, Nelson M, Khokhlova V, Pouget P, Venance L, Bal T and Destexhe A. (2016) Intracellular Impedance Measurements Reveal Non-ohmic Properties of the Extracellular Medium around Neurons. Biophys J. 110: 234-246.

[7] Rall, W. (1962) Electrophysiology of a dendritic neuron model. Biophys J. 2: 145-167.

[8] Rall, W. (1995) The theoretical foundations of dendritic function. MIT Press, Cambridge, MA.

[9] Plonsey, R. (1969) Bioelectric Phenomena. McGrawHill, New York.

[10] Tuckwell, H.C. (1988) Introduction to Theoretical Neurobiology: Linear cable theory and dendritic structure. Cambridge University Press, Cambridge, UK.

[11] Bédard, C. and Destexhe, A. (2013) Generalized cable theory for neurons in complex and heterogeneous media. Physical Review E 88 : 022709.

[12] Bédard, C. and Destexhe A., (2014) Generalized cable formalism to calculate the magnetic field of single neurons and neuronal populations, Physical Review E 90: 042723,

[13] Bédard, C., Kröger, H. and Destexhe, A. (2004) Modeling extracellular field potentials and the frequency-filtering properties of extracellular space. Biophys. J. 86: 1829-1842.

[14] Bédard, C. and Destexhe, A. (2009) Macroscopic models of local field potentials and the apparent 1/f noise in brain activity. Biophys. J. 96: 2589-2603.

[15] Bedard, C. and Destexhe, A. (2012) Modeling local field potentials and their interaction with the extracellular medium. In: Handbook of Neural Activity Measurement, Edited by Brette R. and Destexhe A., Cambridge University Press, Cambridge, UK, pp. 136-191.

[16] Bédard, C., Kröger, H. and Destexhe, A., (2006b) Model of low-pass filtering of local field potentials in brain tissue. Phys. Rev. E 73: 051911.

[17] Bédard, C. and Destexhe, A. (2011) A generalized theory for current-source density analysis in brain tissue. Physical Review E 84: 041909.

[18] Mitzdorf, U. (1985) Current source-density method and application in cat cerebral cortex: investigation of evoked potentials and EEG phenomena. Physiol. Reviews 65: 37-100.

[19] Pritchard, W.S. (1992) The Brain in fractal time: $1 / f$-like power spectrum scaling of the human electroencephalogram. Int. J. Neurosci. 66: 119-129.

[20] Novikov, E., A. Novikov, D. Shannahoff-Khalsa, B. Schwartz, and J. Wright. (1997) Scale-similar activity in the brain. Phys. Rev. E. 56: R2387-R2389.

[21] Bhattacharya, J., and H. Petsche. (2001) Universality in the brain while listening to music. Proceedings : Biological Sciences. 268 : 2423-2433.

[22] Bédard, C., Kröger, H. and Destexhe, A. (2006) Does the $1 / \mathrm{f}$ frequency scaling of brain signals reflect self-organized critical states ?. Physical Review Lett. 97: 118102.

[23] Dehghani, N, Bédard, C., Cash, S.S., Halgren, E. and Destexhe, A. (2010) Comparative power spectral analysis of simultaneous elecroencephalographic and magnetoencephalographic recordings in humans suggests non-resistive extracellular media. J. Computational Neurosci. 29: 405-421.

[24] Milstein JN and Koch C. (2008) Dynamic moment analysis of the extracellular electric field of a biologically realistic spiking neuron. Neural Comput. 20: 2070-2084.

[25] Jensen, H.J. (1998) Self-Organized Criticality. Emergent Complex Behavior in Physical and Biological 
Systems. Cambridge University Press, Cambridge, UK.

[26] Beggs, J., and Plenz, D. (2003) Neuronal avalanches in neocortical circuits. J. Neurosci.. 23 : 11167-11177.

[27] Dehghani, N., Hatsopoulos, N.G., Haga, Z.D., Parker, R.A., Greger, B., Halgren, E., Cash, S.S. and Destexhe, A. (2012) Avalanche analysis from multi-electrode ensemble recordings in cat, monkey and human cerebral cortex during wakefulness and sleep. Frontiers Physiol. 3: 302.

[28] Pettersen, K.H. and Einevoll, G.T. (2008) Amplitude variability and extracellular low-pass filtering of neuronal spikes. Biophys J. 94: 784-802.

[29] Diard, J-P., Le Gorrec, B. and Montella, C. (1999) Linear diffusion impedance. General expression and applications. J. Electroanalytical Chem. . 471: 126-131.

[30] Taylor, S.R. and Gileadi, E. (1995) The physical interpretation of the Warburg impedance. Corrosion
51:

[31] Bédard, C., Rodrigues, S., Roy, N., Contreras, D. and Destexhe, A. (2010) Evidence for frequencydependent extracellular impedance from the transfer function between extracellular and intracellular potentials. J. Computational Neurosci. 29: 389403.

[32] Hille B. (1992) Ionic Channels of Excitable Membranes. 2nd Ed., Sinauer Associates Inc., Sunderland,Massachusetts. 210 (2012) 3-14.

[33] Bédard, Claude and Destexhe, Alain, (2014) MeanField Formulation of Maxwell Equations to Model Electrically Inhomogeneous and Isotropic Media, Journal of Electromagnetic Analysis and Applications, 6, 10, p. 296,

[34] Smirnov V. (1970) A Course of Higher Mathematics, Volume III, Part. 2 chap 6, MIR Editions, Moscow. 